\title{
A Prospective Study on the Efficiency of Ciprofloxacin in Combination with Chloramphenicol against Multiple Antibiotics Resistant Klebsiella pneumoniae
}

\author{
Akash Ahmed1, Ishrat Binte Aftab', Tonima Fairooz Mouly', Sinthia Kabir Mumu1, \\ Abu Syed Md. Mosaddek ${ }^{2}$, Mahboob Hossain ${ }^{*}$
}

${ }^{1}$ Microbiology Program, Department of Mathematics and Natural Sciences, BRAC University, Dhaka, Bangladesh

${ }^{2}$ Department of Pharmacology, Uttara Adhunik Medical College, Dhaka, Bangladesh

Email: ^mmhossain@bracu.ac.bd

How to cite this paper: Ahmed, A., Aftab, I.B., Mouly, T.F., Mumu, S.K., Mosaddek, A.S.M. and Hossain, M. (2020) A Prospective Study on the Efficiency of Ciprofloxacin in Combination with Chloramphenicol against Multiple Antibiotics Resistant Klebsiella pneumoniae. Advances in Microbiology, 10, 411-421. https://doi.org/10.4236/aim.2020.109030

Received: February 23, 2020

Accepted: September 8, 2020

Published: September 11, 2020

Copyright ( 2020 by author(s) and Scientific Research Publishing Inc. This work is licensed under the Creative Commons Attribution International License (CC BY 4.0).

http://creativecommons.org/licenses/by/4.0/ (c) (i) Open Access

\begin{abstract}
Pneumonia is the single largest infectious cause of death in children worldwide and also a form of an acute respiratory infection that affects the lung. The purpose of the study was to develop a new approach to treat antibiotic-resistant $K$. pneumoniae infection. This study aimed in quest of a drug to combine with ciprofloxacin, a broad spectrum antibiotic frequently used to treat lung infections. Methodology: A total of 23 lung infection bacterial samples were collected and studied against 14 antibiotics of different classes. The disk diffusion method was performed to determine synergy screening, MIC value, and qualitative toxicity analysis of ciprofloxacin and chloramphenicol combination. Results: After primary screening of antibiotic susceptibility, they were categorized into multidrug-resistant (MDR), extensively drug-resistant (XDR) and pan drug-resistant (PDR) pathogens where 9 isolates were MDR, 5 were XDR and 3 isolates were PDR. Furthermore, they were trialed in combination ciprofloxacin along with other 7 drugs in disk diffusion to explore the synergistic effect. The combination of ciprofloxacin and moxifloxacin, ciprofloxacin and chloramphenicol were found to be synergic. Then the MIC test was done for the combination ciprofloxacin and chloramphenicol. When the MIC result was generated, the MIC of the respective combination was analyzed. Furthermore, the fractional inhibitory concentration (FIC) was calculated and in accordance with the results of the FIC index, ciprofloxacin-chloramphenicol combination has shown value 0.4510 which revealed a synergistic effect against multi-drug resistant Klebsiella pneumoniae. Conclusion: Given these points, if the efficiency of this an-
\end{abstract}


tibiotic can be accelerated from combination with other drugs, it might be lifesaving and cost effective as well.

\section{Keywords}

Multidrug Resistant Bacteria, Klebsiella pneumoniae, Combination Therapy, FIC

\section{Introduction}

Pneumonia has become the leading cause of child death since many decades in developing countries [1]. Combating with the Klebsiella pneumoniae is harder when it is resistant to several antibiotics. Undoubtedly, the healthcare system has been updated in each second but it was 1987 last, when a new antibiotic class was introduced. As a result, what will be the future of treating severely antibiotic resistant bacterial infection is a burning question in front of the modern science [2]. Different definitions for multidrug-resistant (MDR), extensively drug-resistant (XDR) and pan drug-resistant (PDR) bacteria are being used in the medical literature to characterize the different patterns of resistance found in healthcare-associated antimicrobial resistant bacteria [3].

The emergence of antibiotic resistant microorganism is natural and genetically over time. Still there are certain acceleration factors yielding the development of resistant strain. According to World Health Organization (WHO) the overuse and misuse of frequent antibiotic is the culprit behind the resistant mechanism [4]. Further, researchers explored poor infection control, inadequate sanitary conditions and inappropriate food-handling encourage the spread of antimicrobial resistance. Particularly, the developing country like Bangladesh is greatly threatened by the emergence of extremely drug-resistant bacteria. It is figured out that up to 86 percent of antibiotics are consumed without the prescription, resulting misuse of conventional and modern antibiotic as well [5].

Klebsiella pneumoniae carbapenemases (KPCs) were identified in the USA in 1996 [6]. The KPC is produced by the Klebsiella pneumoniae to resist the antibiotic class carbapenem. The mortality among patients infected with KPC is high, as a result of the limited antibiotic options remaining (often colistin, tigecycline, or aminoglycosides). Triple drug combinations using colistin, tigecycline, and imipenem have recently been associated with improved survival among patients with bacteraemia [7]. Klebsiella pneumoniae is one of the MDR organisms claimed as a serious danger to health by the World Health Organization, the US Centers for Disease Control and Prevention and the UK Department of Health [8]. Researchers point out the emergence of colistin resistance in MDR $K$. pneumoniae rising from the mutations of the mgrB gene, a negative regulator of the PhoPQ signalling system [9]. The PhoPQ component system is a regulator of envelope remodelling, predominantly the lipopolysaccharide (LPS) lipid, a section, and subsidizes to bacterial resistance to innate immune killing [10]. $K$. 
pneumoniae PhoPQ also manages lipid a plasticity in vivo and in vitro resulting developing resistant mechanism [11].

Ciprofloxacin consists of 8 different functional groups (shown in Figure 1). It is a broad spectrum antibiotic against mostly gram negative bacteria. Ciprofloxacin combination with metronidazole is one of several first-line antibiotic regimens recommended by the Infectious Diseases Society of America for the treatment of community-acquired abdominal infections in adults [12]. Gradually, ciprofloxacin is effective in combating bone and joint infection with the treatment of sinusitis [13].

However, physicians start using timely antibiotic combination therapy to improve the patient survival since the antibiotic treatment for these resistant bacteria is limited [14]. Hence the new antibiotic class is not generating for a long time, in the era of rising antimicrobial resistance, coupled with a continued dwindling pipeline of drugs to treat these infections [15]. It is evident that combination therapy will be the most suitable advantage to treat resistant Klebsiella pneumoniae.

\section{Materials and Methods}

The experiment was carried in the laboratory of BRAC University to study the activity of ciprofloxacin having combination with several drugs against multidrug resistant Klebsiella pneumoniae. The experiment proceed mainly involved in collecting the pathogenic Klebsiella pneumoniae and their antibiotic susceptibility testing. Then, the samples were categorized into Multidrug resistant (MDR), Extensively Drug resistant (XDR) and Pan Drug resistant (PDR) on the basis of their susceptibility to 14 different antibiotics including Penicillin G, Penicillin V, Ampicillin, Amoxicillin, Cefixime, Imipenem, Cephalosporin (Cell wall synthesis inhibitor); Chloramphenicol, Tetracycline, Azithromycin (Protein synthesis inhibitor) and Ciprofloxacin, Nalidixic Acid, Rifampicin and Moxifloxacin (Nucleic acid synthesis inhibitor). Randomly 2 MDR, 2 XDR and 2 PDR pathogen are picked for the activity study of several drugs combined with ciprofloxacin. In this study, 8 different drugs of various groups are combined with ciprofloxacin disc to demonstrate their combined effect. The combination of chloramphenicol with ciprofloxacin is assumed to be efficient from exploring the zone of inhibition data result. To be clear with the assumption, the Minimum Inhibitory Concentration (MIC) of these combinations was figured out along with the MIC of individual one. Finally, the Fractional Inhibitory Concentration (FIC) index of each combination (Except the combination of ciprofloxacin and probiotic) was calculated and compared with the standard for statistical validation.

\subsection{Collection of Pathogenic Klebsiella pneumoniae}

Clinically identified Klebsiella pneumoniae was collected from the National Institute of Diseases of the Chest and Hospital (NIDCH) and microbiology department of Uttara Adhunik Medical College Hospital (UAMCH).The isolates of 

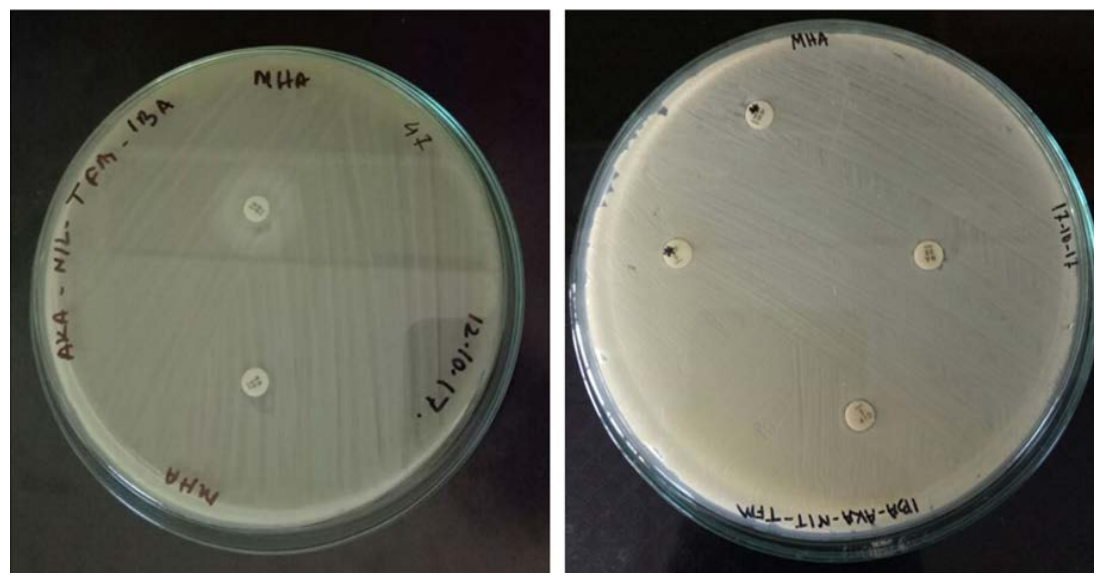

Figure 1. The antibiotic susceptibility test result of Klebsiella pneumoniae.

Klebsiella pneumoniae was sub-cultured to nutrient agar slant and carried to BRAC University laboratory. The nutrient agar slant was incubated at $37^{\circ} \mathrm{C}$ for 24 hours. Then the pathogen was transferred to nutrient agar plate by streaking plate method.

At the same time these samples are stored at $-20^{\circ} \mathrm{C}$ in glycerol media as stock.

\subsection{Collection of Antibiotics and Drugs for Combination}

Ciprofloxacin was taken from the product of Square Pharmaceuticals, Ciprocin $500 \mathrm{mg}$. The other drugs for combination purpose are listed in Table 1.

\subsection{Preparation of Stock Solution of Drugs and Antibiotics}

Commercially available tablet or capsule was bought and dissolved in $10 \mathrm{ml}$ physiological saline. Though the excipients of those drugs were also inside the solution, it was considered not to be interfered with the desired product since excipients are chemically inert.

Tablet or capsule was poured in physiological saline. As a result, the volume of the $10 \mathrm{ml}$ saline raised very little which was negligible and excluded from the calculation.

\subsection{Disc Diffusion Method}

Agar surface of Muller-Hinton Agar plate was streaked by a sterile cotton swab with the collected pathogenic Klebsiella pneumoniae strain from the physiological saline which was compared with McFarland standard 1 solution. McFarland standard 1 solution shows the density of $3 \times 10^{8} \mathrm{CFU}$ (Colony Forming Unit) per ml. Antibiotics discs were placed on solidified agar plates at equal distance apart. The plates were kept standby for $10 \mathrm{~min}$. Then the plates were incubated at $37^{\circ} \mathrm{C}$ for 24 hours. The disc diffusion test was done to determine the antibiotic resistant pattern of the pathogens as well as to categorize to MDR, XDR and PDR by the guideline of Clinical and Laboratory Standards Institute (CLSI). Around 14 antibiotic discs were used in this study. 
Table 1. The list of drugs used for combination.

\begin{tabular}{cccc}
\hline Trade Name & Company Name & Generic Name & Class \\
\hline Fexo $120 \mathrm{mg}$ & Square Pharma & Fexofenadine & Antihistamine \\
Rifagut $200 \mathrm{mg}$ & Opsonin Pharma & Rifaximin & Miscellaneous Antibiotic \\
Indever $10 \mathrm{mg}$ & ACI Limited & Propranol Hydrochloride & Calcium channel blocker \\
Cloram $5 \mathrm{mg} / \mathrm{ml}$ & Ibn Sina Pharma. & Chloramphenicol & Antibiotic \\
Tycil $500 \mathrm{mg}$ & Beximco Pharma & Amoxicillin & Antibiotic \\
Moxibac & Popular Pharma & Moxifloxacin & Antibiotic \\
Ciprocin & Square Pharma & Ciprofloxacin & Antibiotic \\
\hline
\end{tabular}

\subsection{Determining the Minimum Inhibitory Concentration (MIC) of Antibiotics}

The study was designed to observe the efficiency of ciprofloxacin alone and with combination against highly antibiotic resistant Klebsiella pneumoniae. Hence, the MIC of ciprofloxacin and the MIC of other individual antibiotics were figured out with or without combination.

Determining the MIC, different concentration of antibiotic was required. For this purpose, serial dilution was carried out with the aid of Brain Heart Infusion (BHI) broth as diluent. From the C1V1 $=\mathrm{C} 2 \mathrm{~V} 2$ formula, desired concentration was prepared by the addition of stock solution to BHI broth.

A wide range dilution was prepared with individual antibiotics and combination as well. Throughout the study, it was practiced to keep the concentration of different compounds same. Each test tube having a known concentration of antibiotic was inoculated with $100 \mu \mathrm{L}$ of McFarland 1 standard pathogenic suspension and kept at 370 Celsius for 24 hours. The next day, each tube was critically observed to identify either turbid or clear. The lowest concentration of antibiotic gave clear tube was considered as MIC value.

The MIC test was done twice with different dilution range to get more sophisticated result through arithmetic mean calculation.

\subsection{Determining the Fractional Inhibitory Concentration (FIC) Index}

Fractional Inhibitory Concentration (FIC) index is a statistical tool for validation. The standard value of FIC index is 0.5 to 4 . The lower value represents the synergism and higher value for antagonism.

To get FIC index, firstly, FIC was calculated by the following equation:

$$
\text { FIC }=\frac{\text { MIC of the agents in combination }}{\text { MIC of the agent alone }}
$$

FIC index was calculated by the formula of:

$$
\text { FIC Index }=\sum \frac{\text { MIC of the agents in combination }}{\text { MIC of the agent alone }}
$$


Synergy is defined as $\Sigma F I C \leq 0.5$, indifference is defined as $0.5<\Sigma F I C \leq 4$, and antagonism is defined as $\mathrm{EIC}>4[16]$.

The average FIC index from six Klebsiella pneumoniae was determined and compared to standard.

\section{Results}

\subsection{Categorizing the Pathogenic Klebsiella pneumoniae}

When a species of microorganism shows non-susceptibility to at least one agent in three or more antimicrobial categories it is classified as MDR. When this non-susceptibility is shown against at least one agent in all but two or fewer antimicrobial categories it is classified as XDR. PDR is defined when non-susceptibility is shown to all agents in all antimicrobial categories Table 2

MDR = Resistant to Penicillin G, Penicillin V, Ampicillin, Amoxicillin, Ciprofloxacin, Nalidixic acid, Chloramphenicol.

$\mathrm{XDR}=$ Resistant to Penicillin G, Penicillin V, Ampicillin, Amoxicillin, Cephalosporin, Cefixime, Chloramphenicol, Tetracycline, Nalidixic acid, Ciprofloxacin.

PDR $=$ Resistant to all 14 antibiotics.

A. The antibiotic susceptibility test result of Ciprofloxacin + Amoxicillin combination against Klebsiella pneumoniae (left picture). B. The antibiotic susceptibility test result of Ciprofloxacin + Rifaximin and Ciprofloxacin + Fexofenadine combination against Klebsiella pneumoniae (right picture) shown in Figure 1.

\subsection{Screening Antibiotic Combination against MDR, XDR and PDR Klebsiella pneumoniae}

Two MDR, two XDR and two PDR were selected randomly from the collected Klebsiella pneumoniae pathogen in an effort to inhibit the growth of the pathogen by ciprofloxacin having various combinations with 7 different drugs including antihistamine, antibiotic, calcium channel blocker and probiotic which are shown in Table 3.

\subsection{Determination of the Arithmetic Mean MIC of Ciprofloxacin, Chloramphenicol and the Combination of Ciprofloxacin and Chloramphenicol}

Since these pathogens were multidrug resistant, the MIC value of ciprofloxacin and chloramphenicol was pretty high and the MIC value of the combination was nearly low as shown in Table 4.

\subsection{The Average FIC Index (MDR, XDR, PDR) of Ciprofloxacin and Chloramphenicol}

The arithmetic mean of FIC index is 0.4510 which is less than 0.5 yielding statistical significant synergistic effect of ciprofloxacin and chloramphenicol against the multiple antibiotics resistant Klebsiella pneumoniae. 
Table 2. The number of MDR, XDR and PDR Klebsiella pneumoniae.

\begin{tabular}{cccc}
\hline Total Sample & MDR & XDR & PDR \\
\hline 23 & 9 & 5 & 3 \\
\hline
\end{tabular}

Table 3. The combination of ciprofloxacin with several drugs and the synergy screening.

\begin{tabular}{cc}
\hline Combination of Drugs & Inhibition of Growth \\
\hline Ciprofloxacin + Chloramphenicol & + \\
Ciprofloxacin + Amoxicillin & - \\
Ciprofloxacin + Rifaximin & - \\
Ciprofloxacin + Propranol Hydrochloride & - \\
Ciprofloxacin + Fexofenadine & + \\
Ciprofloxacin + Moxifloxacin & - \\
Ciprofloxacin + Clonazipum & - \\
\hline
\end{tabular}

Key: $(+)$ = synergic, $(-)=$ No change.

Table 4. The Average MIC value of ciprofloxacin, chloramphenicol and their combination in $\mu \mathrm{g} / \mathrm{ml} \&$ FIC Index.

\begin{tabular}{cccccc}
\hline \multirow{2}{*}{ Category } & Sample & \multicolumn{4}{c}{ MIC (in $\mu \mathrm{m} / \mathrm{ml})$} \\
\cline { 3 - 5 } & Number & $\begin{array}{c}\text { Ciprofloxaci } \\
\text { Only }\end{array}$ & $\begin{array}{c}\text { Chloramphenicol } \\
\text { Only }\end{array}$ & $\begin{array}{c}\text { Ciprofloxacin }+ \\
\text { Chloramphenicol }\end{array}$ & FIC Index \\
\hline \multirow{2}{*}{ MDR } & Sample 2 & 425 & 225 & 56.25 & 0.3823 \\
& Sample 27 & 225 & 112.5 & 12.81 & 0.1708 \\
\multirow{2}{*}{ XDR } & Sample 8 & 450 & 56.25 & 31.25 & 0.5625 \\
& Sample 42 & 225 & 250 & 68.75 & 0.5805 \\
\multirow{2}{*}{ PDR } & Sample 1 & 475 & 250 & 56.25 & 0.3434 \\
& Sample 3 & 450 & 225 & 100 & 0.6666 \\
\hline
\end{tabular}

Key: MIC = Minimum Inhibitory Concentration, $\mathrm{MDR}=$ Multidrug Resistant, XDR = Extensively Drug Resistant, PDR = Pan Drug Resistant, FIC = Fractional Inhibitory Concentration.

\section{Discussion}

Antibiotic resistant bacterial infection is becoming the greatest threat to mankind as mentioned earlier, commercially available antibiotics are being challenged by the pathogen as well. The United States Centers for Disease Control and Prevention estimate the infections caused by antibiotic-resistant bacteria result in some two million cases of illness and 23,000 deaths in the U.S. annually. Additionally, the European Centre for Disease Prevention and Control produces similar numbers, estimating that antibiotic-resistant bacteria kill approximately 25,000 Europeans every year [17].

This study clearly presents the emerging antibiotic resistant Klebsiella pneumoniae resistant pattern. It is noted that, out of 23 samples 17 of the bacteria were antibiotic resistant which is definitely challenging to medical science. A recent study figured out that a woman died in September, 2016 at Nevada, USA was infected with Klebsiella bacteria which were resistant to 26 different antibio- 
tics. Indeed, the bacteria were resistant to all available antimicrobial drugs in the USA reported by The US Centre for Disease Control and Prevention [18].

It was found that one of the major factors of increasing antibiotic resistance is the overuse and misuse of antibiotics which is common practice in the countries like India, Pakistan, Bangladesh and Sri Lanka [18]. Since the pathogen is emerging with the ability to survive within antibiotic treatment and new antibiotics are not developed, treatment procedure attracts the combination available antibiotic and herbal extracts. It has been noted that combination therapy may often be necessary for successful patient outcomes, but data in humans are still lacking and are often limited by retrospective and non-comparative study designs [19]. This study only finds the in vitro synergistic activities of ciprofloxacin and chloramphenicol against immensely drug resistant Klebsiella pneumoniae which should be followed up from in vivo modeling.

Further, antibiotic resistant Klebsiella pneumoniae has been considered as a major threat to global healthcare system. As a result, numerous antibiotic combination studies have been developed against this pathogen. The combination of rifampin and colistin has been found to be bactericidal in KPC-producing $K$. pneumoniae isolates [20]. Again, the combination of rifampin, meropenem, and colistin was bactericidal against MBL-producing $K$. pneumoniae found in a study [21]. Tigecycline and meropenem were found to be bactericidal against XDR Klebsiella pneumoniae [22]. Tigecycline and amikacin combination scored 1.25 FIC index on a study against Klebsiella pneumoniae [23].

Again, the combination of doripenem and levofloxacin scored 0.5 FIC index against Klebsiella pneumonia infected ICU patient which represents the synergistic effect of these two antibiotics, the study also documented doripenem and colistin as 0.75 FIC index considering additive activity [24].

This study reveals it's novelty for combination of ciprofloxacin and chloramphenicol which has not been documented yet. It is the first time when the synergistic activity of these two antibiotics has been found in vitro experiment. The use of antibiotics in combination is already a common hospital procedure in empirical treatment of severe infections but the guideline of using the combination has not been well established. Several investigations have explored the use of various combination regimens for highly antibiotic resistant Klebsiella pneumoniae, but these investigations often lack in vivo validation. It remains unknown which combinations of antimicrobial agents/classes are most effective for the treatment of resistant pathogen [22].

Another interesting finding is the FIC index of ciprofloxacin and chloramphenicol found in this study is 0.45 ; compared to literature, the combination of doripenem and levofloxacin scored 0.5, this levofloxacin is from quinolone group other than ciprofloxacin.

\section{Conclusion}

In conclusion, it should not be mistaken to assert the upcoming threat of antibi- 
otic-resistant pathogen, particularly Klebsiella pneumonia,that is emerging as a superbug. A developing country like Bangladesh, is going to face a terrible challenge of these emerging pathogen unless and until the frequent misuse and overuse of antibiotic is abridged. Undoubtedly, the healthcare system due to antibiotic resistant bacterial infection also faces economic penalties as well. However, when the question is about life, new methods must be developed to combat these superbugs and existing antibiotic combination can be a good choice. Nevertheless, ciprofloxacin is well established antibiotic having broad spectrum bactericidal activity. Hence, if the efficiency of this antibiotic can be accelerated from combination with other drugs, it might be lifesaving and cost effective as well. Moreover, developing country like ours' can grab the chance to combat antibiotic resistant Klebsiella pneumoniae from this combination and reduce the mortality rate from prolonged pneumonia since we are endangered floating in the sea of emerging antibiotic resistant pathogen.

\section{Acknowledgements}

Dr. Abu Syeed Mosaddek, professor and head at Pharmacology, Uttara Adhunik Medical College \& Hospital, Dr. Most. Fhamida Begum, professor \& head at Microbiology, Uttara Adhunik Medical College \& Hospital and the authority of National Institute of Diseases of the Chest and Hospital (NIDCH) for giving the opportunity to sample collection.

\section{Conflicts of Interest}

The authors declare no conflicts of interest regarding the publication of this paper.

\section{References}

[1] Saha, S., et al. (2016) Epidemiology and Risk Factors for Pneumonia Severity and Mortality in Bangladeshi Children $<5$ Years of Age before 10-Valent Pneumococcal Conjugate Vaccine Introduction. BMC Public Health, 16, Article No. 1233. https://doi.org/10.1186/s12889-016-3897-9

[2] Groopman, J. (2008) Superbugs, the New Generation of Resistant Infections Is Almost Impossible to Treat.

[3] Magiorakos, A.P., et al. (2012) Multidrug-Resistant, Extensively Drug-Resistant and Pandrug-Resistant Bacteria: An International Expert Proposal for Interim Standard Definitions for Acquired Resistance. Clinical Microbiology and Infection, 18, 268-281. https://doi.org/10.1111/j.1469-0691.2011.03570.x

[4] Togoobaatar, G., et al. (2010) A Survey of Non-Prescribed Use of Antibiotics for Children in an Urban Community in Mongolia. Bulletin of the World Health Organization, 88, 930-936.

[5] Fair, R.J. and Tor, Y. (2014) Antibiotics and Bacterial Resistance in the 21st Century. Perspectives in Medicinal Chemistry, 6, 25-64. https://doi.org/10.4137/PMC.S14459

[6] Yigit, H., et al. (2001) Novel Carbapenem-Hydrolyzing $\beta$-Lactamase, KPC-1, from a Carbapenem-Resistant Strain of Klebsiella pneumonia. Antimicrobial Agents and 
Chemotherapy, 45, 1151-1161. https://doi.org/10.1128/AAC.45.4.1151-1161.2001

[7] Munoz-Price, L.S., et al. (2013) Clinical Epidemiology of the Global Expansion of Klebsiella pneumoniae Carbapenemases. The Lancet Infectious Diseases, 13, 785-796. https://doi.org/10.1016/S1473-3099(13)70190-7

[8] Kidd, T.J., et al. (2017) A Klebsiella pneumoniae Antibiotic Resistance Mechanism That Subdues Host Defences and Promotes Virulence. EMBO Molecular Medicine, 9, 430-447. https://doi.org/10.15252/emmm.201607336

[9] Lippa, A.M. and Goulian, M. (2009) Feedback Inhibition in the PhoQ/PhoP Signaling System by a Membrane Peptide. PLOS Genetics, 5, e1000788.

[10] Groisman, E.A. (2001) The Pleiotropic Two-Component Regulatory System PhoPPhoQ. Journal of Bacteriology, 183, 1835-1842. https://doi.org/10.1128/JB.183.6.1835-1842.2001

[11] Llobet, E., et al. (2015) Deciphering Tissue-Induced Klebsiella pneumoniae Lipid a Structure. Proceedings of the National Academy of Sciences of the United States of America, 112, E6369-E6378. https://doi.org/10.1073/pnas.1508820112

[12] Solomkin, J.S., et al. (2010) Diagnosis and Management of Complicated Intra-Abdominal Infection in Adults and Children: Guidelines by the Surgical Infection Society and the Infectious Diseases Society of America. Clinical Infectious Diseases, 50, 133-164. https://doi.org/10.1086/649554

[13] Osmon, D.R., et al. (2013) Diagnosis and Management of Prosthetic Joint Infection: Clinical Practice Guidelines by the Infectious Diseases Society of America. Clinical Infectious Diseases, 56, e1-e25. https://doi.org/10.1093/cid/cis803

[14] Bush, K. and Fisher, J.F. (2011) Epidemiological Expansion, Structural Studies, and Clinical Challenges of New $\beta$-Lactamases from Gram-Negative Bacteria. Annual Review of Microbiology, 65, 455-478. https://doi.org/10.1146/annurev-micro-090110-102911

[15] Pucci, M.J. and Bush, K. (2013) Investigational Antimicrobial Agents of 2013. Clinical Microbiology Reviews, 26, 792-821. https://doi.org/10.1128/CMR.00033-13

[16] Meletiadis, J., Pournaras, S., Roilides, E. and Walsh, T.J. (2010) Defining Fractional Inhibitory Concentration Index Cutoffs for Additive Interactions Based on Self-Drug Additive Combinations, Monte Carlo Simulation Analysis, and In Vitro-In Vivo Correlation Data for Antifungal Drug Combinations against Aspergillus fumigates. Antimicrobial Agents and Chemotherapy, 54, 602-609.

https://doi.org/10.1128/AAC.00999-09

[17] Boyce, M. (2017) A New Threat to Public Health: Antibiotic-Resistant Bacteria. Voices of DGHI.

[18] Branswell, H. (2017) A Nevada Woman Dies of a Superbug Resistant to Every Available Antibiotic in the US.

[19] Elizabeth, V.H.T. and Hirsch, B. (2010) Detection and Treatment Options for Klebsiella pneumoniae Carbapenemases (KPCs): An Emerging Cause of Multidrug-Resistant Infection. Journal of Antimicrobial Chemotherapy, 65, 1119-1125.

[20] Elemam, A., Rahimian, J. and Doymaz, M. (2010) In Vitro Evaluation of Antibiotic Synergy for Polymyxin B-Resistant Carbapenemase-Producing Klebsiella pneumoniae. Journal of Clinical Microbiology, 48, 3558-3562.

https://doi.org/10.1128/JCM.01106-10

[21] Tängdén, T., Hickman, R.A., Forsberg, P., Lagerbäck, P., Giske, C.G. and Cars, O. (2014) Evaluation of Double- and Triple-Antibiotic Combinations for VIM- and NDM-Producing Klebsiella pneumoniae by in Vitro Time-Kill Experiments. Anti- 
microbial Agents and Chemotherapy, 58, 1757-1762.

https://doi.org/10.1128/AAC.00741-13

[22] Lim, T.P., et al. (2015) In Vitro Pharmacodynamics of Various Antibiotics in Combination against Extensively Drug-Resistant Klebsiella pneumonia. Antimicrobial Agents and Chemotherapy, 59, 2515-2524.

[23] Humphries, R.M., Kelesidis, T., Bard, J.D., Ward, K.W., Bhattacharya, D. and Lewinski, M.A. (2010) Successful Treatment of Pan-Resistant Klebsiella pneumoniae Pneumonia and Bacteraemia with a Combination of High-Dose Tigecycline and Colistin. Journal of Clinical Microbiology, 59, 1383-1386.

https://doi.org/10.1099/jmm.0.023010-0

[24] Celik, B. O., Mataraci-Kara, E. and Yilmaz, M. (2014) Effects of Various Antibiotics Alone or in Combination with Doripenem against Klebsiella pneumoniae Strains Isolated in an Intensive Care Unit. BioMed Research International, 2014, Article ID: 397421. https://doi.org/10.1155/2014/397421 